\title{
Draft genome sequences of three NDM-1-producing Enterobacteriaceae species isolated from Brazil
}

\author{
Polyana Silva Pereira', Rodolpho Mattos Albano², \\ Marise Dutra Asensi ${ }^{1}$, Ana Paula D'Alincourt Carvalho-Assef $1 /{ }^{1+}$ \\ 'Laboratório de Pesquisa em Infecção Hospitalar, Instituto Oswaldo Cruz-Fiocruz, Rio de Janeiro, RJ, Brasil \\ 2Departamento de Bioquímica, Universidade do Estado do Rio de Janeiro, Rio de Janeiro, RJ, Brasil
}

\begin{abstract}
The emergence of multidrug-resistant Enterobacteriaceae strains producing carbapenemases, such as NDM-1, has become a major public health issue due to a high dissemination capacity and limited treatment options. Here we describe the draft genome of three NDM-1-producing isolates: Providencia rettgeri (CCBH11880), Enterobacter hormaechei subsp. oharae (CCBH10892) and Klebsiella pneumoniae (CCBH13327), isolated in Brazil. Besides bla $_{N D M-\text { p }}$ resistance genes to aminoglycosides [aadA1, aadA2, aac(6')-Ib-cr] and quinolones (qnrA1, qnrB4) were observed which contributed to the multidrug resistance profile. The element ISAba125 was found associated to the $\mathrm{bla}_{N D M-1}$ gene in all strains.
\end{abstract}

Key words: NDM-1 - Brazil - Enterobacteriaceae

The emergence of carbapenemase-producing Enterobacteriaceae has become a major public health issue worldwide due to a high dissemination capacity and limited treatment options (Nordaman et al. 2011). NDM is a metallo-beta-lactamase first reported in 2009 (Yong et al. 2009) and, now, it has already been detected in several countries worldwide. In Brazil, this carbapenemase was first described in a Providencia rettgeri isolate from the city of Porto Alegre, state of Rio Grande do Sul (RS) (South Region of Brazil), in 2013 (Carvalho-Assef et al. 2013). Then, the detection of six clonally related NDM-producing Enterobacter hormaechei subsp. oharae isolates was reported from the same public hospital (Carvalho-Assef et al. 2014). After that, nine NDM-1-producing Enterobacter cloacae complex isolates and two Morganella morgannii were also recovered from three different hospitals in Porto Alegre (Rozales et al. 2014). Recently, NDM-1-producing bacteria have been described in the state of Rio de Janeiro (RJ) (Southeast Region of Brazil) (Pereira et al. 2014). In 2014, a NDM-1-producing Acinetobacter baumannii was also detected in Londrina, state of Paraná (South Region of Brazil) (Pillonetto et al. 2014).

Here, we aim to announce the draft genome of three NDM-1-producing isolates: $P$. rettgeri (CCBH11880), isolated from a surgical wound of a patient from RS, $E$. hormaechei subsp. oharae (CCBH10892), isolated from a rectal swab of another patient in RS, and Klebsiella pneumoniae (CCBH13327), isolated from a rectal swab of a patient in the city of Rio de Janeiro, RJ.

doi: 10.1590/0074-02760150081

Financial support: CNPq, FIOCRUZ

+ Corresponding author: anapdca@ioc.fiocruz.br

Received 27 February 2015

Accepted 27 April 2015
Initially all of the strains were tested against different antimicrobial drugs and showed multidrug resistance profiles. Genomic DNA of all strains was extracted using QIAamp DNA Blood Mini Kit (Qiagen, Germany). Whole genome shotgun libraries from each strain were prepared with the Nextera XT DNA Sample Prep kit (Illumina Inc, USA), according to the manufacturer's instructions, and sequenced on an Illumina MiSeq system with the MiSeq Reagent v.2 500 cycles kit. Sequence reads were then trimmed and filtered using a Phred score $>20$. The software Geneious v.6.1.7 (Biomatters Ltd, New Zealand) was used to perform de novo assembling. Rapid Annotation using System Technology v.2.0 server was used for genome annotation. Acquired resistance genes were analysed using the ResFinder platform (genomicepidemiology.org). The detailed features of all isolates can be found on Table.

For the $P$. rettgeri isolate (CCBH11880) we obtained 656,560 paired end reads of 250 base pairs (bp), which were assembled into 80 contigs. The $\mathrm{G}+\mathrm{C}$ content for this strain was $41 \%$, considered common for this species. The estimated genome size, comprising all contigs, was 4,999,177 bp. Overall, 4,670 protein coding sequences were found and 89 RNAs were annotated (79 tRNA and 10 rRNA). Acquired resistance genes were searched using the ResFinder platform and different resistance genes were observed such as: aadAl (GenBank JSEQ01000006.1; 199,767-200,555 bp), strA (GenBank JSEQ01000017.1; 91,398-92,201bp), strB (GenBank JSEQ01000017.1; 92,201-93,037 bp), aadB (GenBank JSEQ01000041.1; 326-859 bp), aac(6')-Ib (GenBank JSEQ01000017.1; 2,882-3,400 bp), qnrD (GenBank JSEQ01000028.1; 1,671-2,315 bp), bla ${ }_{\text {OXA-10 }}$ (GenBank JSEQ01000017.1; 2,014-2,814 bp), bla $a_{\mathrm{NDM}-1}$ (GenBank JSEQ01000024.1; 412-1,224 bp), ere(A) (GenBank JSEQ01000025.1; 295-1,521 bp), $m s r(E)$ (GenBank JSEQ01000023.1; 37,090-38,565 bp), $m p h(E)$ (GenBank JSEQ01000023.1; 36,150-37,034 bp), floR (GenBank JSEQ01000017.1; 96,173-97,386 bp), catA1 (GenBank JSEQ01000031.1; 870-1,529 bp), sull (GenBank JSEQ01000017.1; 3,823-4,749 bp), sul2 (GenBank 
TABLE

Genetic information about three NDM-1-producing isolates from Brazil

\begin{tabular}{|c|c|c|c|}
\hline Isolate feature & $\begin{array}{l}\text { Providencia rettgerii } \\
(\mathrm{CCBH} 11880)\end{array}$ & $\begin{array}{c}\text { Enterobacter hormaechei } \\
\text { (CCBH10892) }\end{array}$ & $\begin{array}{l}\text { Klebsiella pneumoniae } \\
\text { (CСBH13327) }\end{array}$ \\
\hline NCBI acession & JSEQ00000000 & JSBO00000000 & JSER00000000 \\
\hline BioProject & PRJNA264579 & PRJNA264581 & PRJNA264954 \\
\hline Isolation source & Surgical wound & Rectal swab & Rectal swab \\
\hline City/state of origin & Porto Alegre/RS & Porto Alegre/RS & Rio de Janeiro/RJ \\
\hline GC content $(\%)$ & 41 & 54.5 & 56.6 \\
\hline Paired end reads (n) & 656,560 & $2,283,589$ & $1,748,579$ \\
\hline Genome coverage & $32 X$ & $106 X$ & $72 \mathrm{X}$ \\
\hline Estimated genome size (bp) & $4,999,177$ & $5,373,562$ & $6,023,847$ \\
\hline Contigs (n) & 80 & 58 & 106 \\
\hline N50 & 282.487 & 277.989 & 133.213 \\
\hline Coding sequences (n) & 4,670 & 5,134 & 5,722 \\
\hline RNAs (n) & 89 & 102 & 99 \\
\hline tRNA (n) & 79 & 89 & 86 \\
\hline rRNA (n) & 10 & 13 & 13 \\
\hline Resistance genes & $\begin{array}{c}\text { aadA1, strA, strB, aadB, } \\
\operatorname{aac}\left(6^{\prime}\right)-I b, \operatorname{qnr} D, \text { bla } \\
\operatorname{ere}(A), \operatorname{msr}(E), \operatorname{mph}(E), \text { floR } \\
\operatorname{cat} 1, \operatorname{sull}, \operatorname{sul} 2, \operatorname{tet}(A), \\
\text { dfrAl, dfrAs }\end{array}$ & 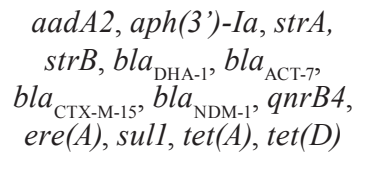 & 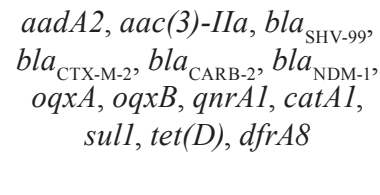 \\
\hline
\end{tabular}

bp: base pair; NCBI: National Center for Biotechnology Information; RJ: state of Rio de Janeiro; RS: state of Rio Grande do Sul.

JSEQ01000017.1; 90,522-91,337 bp), tet(A) (GenBank JSEQ01000017.1; 94,373-95,544 bp), dfrAl (GenBank JSEQ01000006.1; 198,617-199,090 bp) and $d$ frA8 (GenBank JSEQ01000033.1; 334-843 bp).

Genome sequencing of $E$. hormaechei subsp. oharae isolate (CCBH10892) generated 2,283,589 paired end reads of $250 \mathrm{bp}$, yielding 58 contigs after assembly (Geneious v.6.1.7) for a genome size estimative of 5,373,562 bp. A total of 5,134 protein coding sequences and 102 RNAs were observed, being 89 tRNA and 13 rRNA. ResFinder analysis provided the following acquired resistance genes: aadA2 (GenBank JSBO01000022.1; 2,498-3,289 bp), $\operatorname{aph}\left(3^{\prime}\right)-I a$ (GenBank JSBO01000057.1; 354-932 bp), strA (GenBank JSBO01000042.1; 2,178-2,981 bp), strB (GenBank JSBO01000042.1; 2,981-3,817 bp), bla (GenBank JSBO01000036.1; 13,727-14,866 bp), bla ${ }_{\text {ACT-7 }}$ (GenBank JSBO01000001.1; 594,635-595,780 bp), bla $a_{\text {СтХ-м-15 }}$ (GenBank JSBO01000007.1; 210,876-211,751 bp), bla ${ }_{\text {NDM-1 }}$ (GenBank JSBO01000041.1; 4,501-5,313 bp), qnrB4 (GenBank JSBO01000036.1; 8,959-9,606 bp), ere $(A)$ (GenBank JSBO01000002.1; 405,895-406,937 bp), sull (GenBank JSBO01000022.1; 3,707-4,633 bp), tet(A) (GenBank JSBO01000046.1; 2,098-3,297 bp) and tet(D) (GenBank JSBO01000044.1; 1,151-2,335 bp).

The $K$. pneumoniae isolate (CCB13327) had an estimated genome size of $6,023,847 \mathrm{bp}$. An assembly (Geneious v.6.1.7) with 106 contigs was achieved with $1,748,579$ paired end reads of $250 \mathrm{bp}$. A total of 5,722 protein coding sequences were observed, including 99 RNA sequences (86 tRNA and 13 rRNA). Ac- quired resistant genes found were: aadA2 (GenBank JSER01000014.1; 552-1,343 bp), aac(3)-IIa (GenBank JSER01000058.1; 14,738-15,598 bp), bla ${ }_{\mathrm{SHV}-99}$ (GenBank JSER01000008.1; 101,044-101,904 bp), bla (GenBank JSER01000045.1; 2,516-3,391 bp), bla ${ }_{\text {САRВ-2 }}$ (GenBank JSER01000014.1; 1,461-2,375 bp), bla NDM-1 GenBank JSER01000063.1; 4,749-5,561 bp), oqxA (GenBank JSER01000088.1; 4,991-6,166 bp), oqxB (GenBank JSER01000088.1; 2,518-4,967 bp), qnrA1 (GenBank JSER01000080.1; 416-1,072 bp), catA1 (GenBank JSER01000041.1; 3,303-3,962 bp), sull (GenBank JSER01000058.1; 4,497-5,423 bp), tet(D) (GenBank JSER01000091.1; 392-1,576 bp) and dfrA8 (GenBank JSER01000058.1; 816-1,325 bp). Overall, we observed that these NDM-1-producing Enterobacteriaceae strains carry resistance genes to different antimicrobial classes, which can explain the multidrug resistant profile observed.

In P. rettgeri $\mathrm{CCBH} 11880$, the bla ${ }_{\mathrm{NDM}-1}$ was found inside the Tn 125 transposon, which is composed of two copies of ISAba125 (Carvalho-Assef et al. 2013) (data not shown). In the E. hormaechei (Carvalho-Assef et al. 2014) (GenBank acession NG041719) and $K$. pneumoniae isolates, the $b l a_{\mathrm{NDM}-1}$ gene was found flanked by only one copy of IS $A$ ba125 (truncated) at the right boundary (data not shown).

The announcement of the whole-genome sequence of the three NDM-1-producing Enterobacteriaceae strains also provides basis for other studies, which will certainly increase our understanding of the role of this species in the drug resistance scenario. 
Nucleotide sequence accessions - These Whole Genome Shotgun project have been deposited in DDBJ/ ENA/GenBank under the accessions JSEQ00000000, JSBO00000000 and JSER00000000 for $P$. rettgerii (CCBH11880), E. hormaechei (CCBH10892) and $K$. pneumoniae (CCBH13327), respectively. The versions described in this paper are the first version (JSEQ00000000, JSBO00000000 and JSER00000000).

\section{REFERENCES}

Carvalho-Assef AP, Pereira PS, Albano RM, Berião GC, Chagas TPG, Timm LN, da Silva RCF, Falci DR, Asensi MD 2013. Isolation of NDM-producing Providencia rettgeri in Brazil. J Antimicrob Chemother 68: 2956-2957.

Carvalho-Assef AP, Pereira PS, Albano RM, Berião GC, Tavares CP, Chagas TP, Marques EA, Timm LN, da Silva RC, Falci DR, Asensi MD 2014. Detection of NDM-1, CTX-M-15 and qnrB4producing Enterobacter hormaechei isolates in Brazil. Antimicrob Agents Chemother 58: 2475-2476.

Nordmann P, Naas T, Poirel L 2011. Global spread of carbapenemaseproducing Enterobacteriaceae. Emerg Infect Dis 17: 1791-1798.
Pereira PS, Borghi M, Albano RM, Berião GC, Lopes JCO, Asensi MD, Carvalho-Assef APD 2014. First description of NDM-1producing Klebsiella pneumoniae in Brazil. 24th European Congress of Clinical Microbiology and Infectious Diseases, P1196, Poster Session V, Worldwide spread of carbapenem resistance. Available from: escmid.org/escmid_library/online_lecture library $/$ ?search $=1 \&$ current_page $=1 \&$ search_term $=$ polyana + silv $\mathrm{a}+$ pereira\&timeperiod $\% 5 \mathrm{~B} \% 5 \mathrm{D}=\mathrm{ly}$.

Pillonetto M, Arend L, Vespero EC, Pelisson M, Chagas TP, Carvalho-Assef AP, Asensi MD 2014. The first report of NDM-1producing Acinetobacter baumannii ST 25 in Brazil. Antimicrob Agents Chemother 58: 7592-7594.

Rozales FP, Ribeiro VB, Magagnin CM, Pagano M, Lutz L, Falci DR, Machado A, Barth AL, Zavascki AP 2014. Emergence of NDM-1-producing Enterobacteriaceae in Porto Alegre, Brazil. Int J Infect Dis 25: 79-81.

Yong D, Toleman MA, Giske CG, Cho HS, Sundman K, Lee K, Walsh TR 2009. Characterization of a new metallo- $\beta$-lactamase gene, $b l a_{\mathrm{NDM}-1}$, and a novel erythromycin esterase gene carried on a unique genetic structure in Klebsiella pneumoniae sequence type 14 from India. Antimicrob Agents Chemother 53: 5046-5054. 